\title{
UCS REVALUATION AND CONDITIONING MODELS OF ACQUIRED FEARS
}

\author{
Graham C. L. Davey \\ Department of Social Science, The City University, London ECIV OHB, England
}

(Received 21 October 1988)

\begin{abstract}
Summary-Recent developments in the study of Pavlovian conditioning in humans have suggested that the strength of a conditioned response (CR) is affected not only by associative processes that link the conditioned stimulus (CS) with the unconditioned stimulus (UCS), but also by processes which lead the individual to revalue the UCS itself. These latter processes can frequently lead to dramatic changes in CR strength independently of any experience with the CS-UCS contingency. This paper discusses a number of ways in which UCS revaluation can be effected in human subjects, and discusses their implications for conditioning models of acquired fears.
\end{abstract}

In the last $20 \mathrm{yr}$ or so, our conception of the nature of conditioning in animals has changed considerably. No longer is Pavlovian conditioning considered to be the mechanistic learning of S-R reflexes, but it is seen to be comprised of a complex interaction of cognitive and information processing capacities. We know this primarily because of the inferential techniques that have been developed over this period which allow us to infer the nature of some of the unobservable processes that underlie Pavlovian conditioning in animals. Two of the most important findings involve the nature of the associations that underlie conditioning and the nature of the cognitive processes that mediate the conditioned response (CR). In particular, one technique, known as the postconditioning stimulus revaluation method (e.g. Rescorla, 1973, 1980; Dickinson, 1980), has suggested that the majority of first-order Pavlovian conditioning in animals is mediated via learnt associations between the conditioned stimulus (CS) and the unconditioned stimulus (UCS). This procedure involves attempting to revalue the UCS for the animal after conditioning has been completed, and it normally consists of three stages: (i) animals are given pairings of CS and UCS until a CR is established. (ii) $S \mathrm{~s}$ are then given off-the-baseline training with the UCS alone in which the UCS is revalued; in appetitive conditioning this can be done by making the food UCS aversive to the animal by aversion learning (e.g. Rescorla, 1980; Holland and Rescorla, 1975; Cleland and Davey, 1982), and in aversive conditioning by habituating the animal to an electric shock UCS (e.g. Rescorla, 1973, 1980). (iii) The animal is subsequently given test presentations of the CS. The logic here is that if the CR is mediated by a CS-UCS association, the revaluation of the UCS will also affect the $C R$; if, however, the $C R$ is mediated by more reflexive $S-R$ associations, then it will not be affected by this manipulation.

In most first-order conditioning studies which have used postconditioning UCS revaluation procedures (both appetitive and aversive) the results have suggested that the animal learns a CS-UCS association, and that the CR is mediated by the animal's evaluation of the UCS (cf. Rescorla, 1980; Dickinson, 1980 for reviews of these studies). In certain other situations, such as some second-order conditioning procedures, $S-R$ associations may be formed (cf. Rescorla, 1977,1980 ), but, by and large, mediation of the CR appears to occur through a learnt association between the CS and UCS in most of the first-order preparations that have been studied.

While implying that animals generally learn CS-UCS associations in first-order Pavlovian conditioning, these results also have other implications for our conception of Pavlovian responding. First, they imply that the CS activates some kind of internal representation of the UCS and it is this representation that mediates the CR. Secondly, they imply that the strength or even the nature of the CR will depend on how that UCS representation is evaluated when it is activated. Thirdly, they imply that the strength of the $\mathrm{CR}$ can be modulated by procedures which lead to the revaluation of the UCS representation independently of any experience with the CS-UCS contingency; that is, CR strength can be modulated relatively independently by both CS-UCS 
associative processes and UCS revaluation processes. As a process that modulates the strength of the CR, UCS revaluation has implications for Pavlovian conditioning in humans.

\section{PROCESSES OF ASSOCIATION AND UCS REVALUATION IN HUMAN CONDITIONING}

Two lines of evidence suggest that humans also learn CS-UCS associations in first-order Pavlovian conditioning. First, a large number of studies have suggested that human $S$ s only exhibit a differential $C R$ at that point in time when they are first able to verbalise the contingencies between CS + and the UCS (see Dawson and Furedy, 1976; Dawson and Schell, 1987, for reviews of these studies). According to most theorists this implies some kind of 'cognitive relational learning' involving the processing of the relationship between CS and UCS (Grings, 1973; Dawson and Furedy, 1976; Dawson, Catania, Schell and Grings, 1979). Secondly, those studies which have used the postconditioning stimulus revaluation method with human $S$ s have all demonstrated that postconditioning devaluation of the UCS affects the strength of the CR-not just in first-order aversive conditioning, but also in second-order aversive conditioning (e.g. Davey, 1983, 1987a; Davey and McKenna, 1983; Davey and Arulampalam, 1981, 1982). This implies that the CR is mediated by a learnt association between CS and UCS, and is affected by moment-to-moment changes in the $S$ 's evaluation of the UCS. Furthermore, the UCS can be revalued independently of any manipulation which affects the strength of the CS-UCS association.

Davey $(1987 \mathrm{a}-\mathrm{c})$ has pointed out that the sensitivity of human $S$ s to UCS revaluation procedures has important implications for our conception of a contemporary model of Pavlovian conditioning in humans, and to any model of Pavlovian conditioning that is applied to psychopathology in general. In particular, it may well be the case that processes of UCS revaluation are significantly more important than associative strength itself in modulating human $\mathrm{CR}$ strength. The reason for suggesting this is that humans appear to be sensitive to a whole variety of processes which can influence UCS revaluation. In nonhuman animals we are aware of only one UCS revaluation process, and that is individual experience with the UCS alone. However, apart from individual experience with the UCS (cf. Davey and McKenna, 1983), UCS revaluation can occur in human $S \mathrm{~s}$ in at least two other ways.

First, through socially or verbally transmitted information about the UCS. For example, in a laboratory experiment human $S$ s can simply be told that on future presentations the UCS will be less intense. If they believe this, then the UCS will be revalued more favourably and a weaker CR elicited on subsequent CS presentations. Alternatively, they may be told that the UCS will no longer be presented at all (the procedure of instructed extinction). $S \mathrm{~s}$ undergoing instructed extinction tend to rate the UCS as being less aversive and exhibit a substantially weakened CR on subsequent CS presentations (e.g. Davey, 1983, 1987a, 1989a, b; Davey and McKenna, 1983).

The second process of UCS revaluation in humans is one we have studied extensively in our laboratory, and relates to the evaluation of self-observed changes in the strength of the CR and UCR, and to processes of response attribution (cf. Davey, 1987a). If UCS revaluation is a primary process that affects CR strength in Pavlovian learning, then one would intuitively assume that individuals would actively search for information that up-dates their evaluation of the UCSespecially if the UCS is aversive and they want to find ways of reducing the anxiety that the UCS has generated. One important source of information which bears some relation to how the individual can evaluate the UCS is their current reaction to either the CS or UCS itself. If, during aversive conditioning, they emit a strong discriminable fear reaction to the CS or UCS, this may lead them in a post hoc fashion to evaluate the UCS as strongly aversive, which would affect subsequent responding accordingly. In a series of studies of the effects of self-observation of responding, results have suggested that (i) when the $C R$ is made more discriminable (by, for example, providing discriminable response feedback), $S$ s actually emit a greater magnitude $C R$ and show a relative resistance to extinction when compared with $S$ s who have poor $C R$ discrimination (Davey, 1987a); (ii) when $S$ s believe they are emitting a strong aversive CR even though they are not (e.g. in a false feedback study), they exhibit a resistance to extinction compared with both attentional control $S$ s, and $S$ s who believe they are emitting only a weak CR (Davey, 1987a); and (iii) $S \mathrm{~s}$ who believe they are emitting a strong $\mathrm{UCR}$ emit a greater magnitude $\mathrm{CR}$ and show a greater 


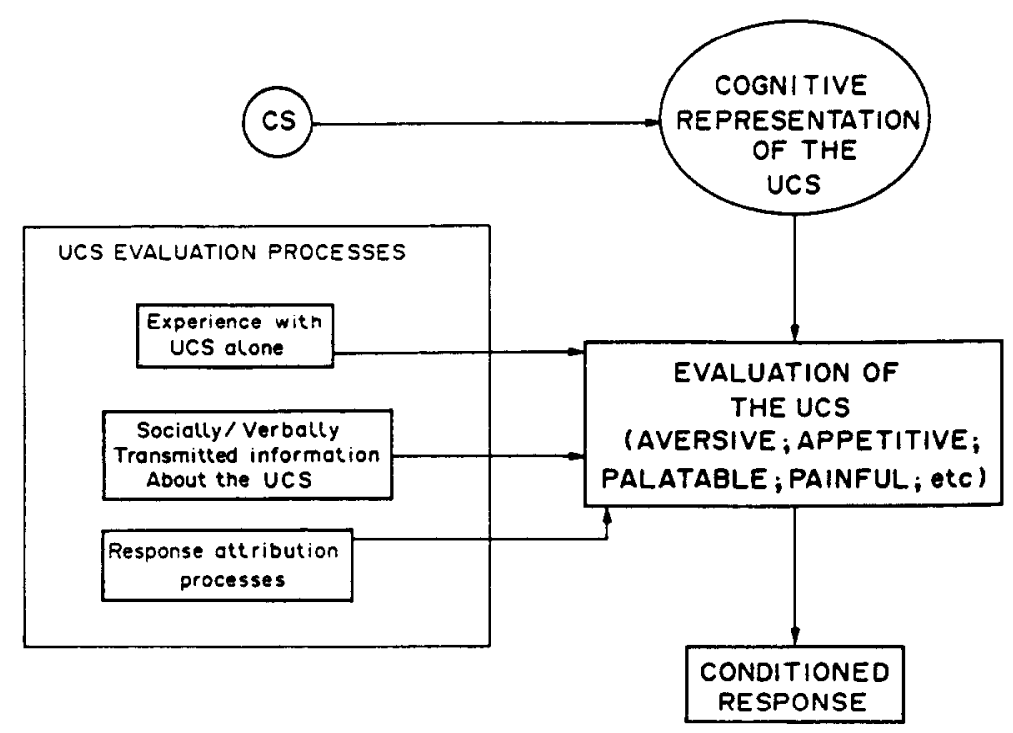

Fig. 1. A schematic representation of the processes involved in modulating the strength of the conditioned response in human Pavlovian conditioning. The CS elicits an internal representation of the UCS which in turn is evaluated for its sensory and affective properties. This evaluation can be influenced by a number of nonassociative processes including individual experience with the UCS alone, socially or verbally transmitted information about the UCS, and self-observation and response attribution processes which provide information derived from the individual's observation of their own conditioned response.

resistance to extinction than $S$ s who believe they are emitting only a weak UCR (even though the actual UCR emitted is similar across groups) (Cracknell and Davey, 1988). Preliminary experimental analysis of these effects has suggested that modulation of CR strength and persistance as a result of the self-observation of responding is mediated via revaluation of the UCS rather than revaluation of either the aversiveness of the CS itself or the $S$ 's own overall state of arousal or 'fearfulness' (cf. Davey, 1988).

\section{A CONTEMPORARY MODEL OF PAVLOVIAN CONDITIONING IN HUMANS}

Figure 1 illustrates the main features of a contemporary model of human conditioning based on our knowledge of the associative substructure that underlies this conditioning, and the processes of UCS revaluation which modulate CR strength. In this model, the CS, through its learnt association with the UCS, comes to elicit a cognitive representation of the UCS. This representation of the UCS is then evaluated, and the resultant evaluation determines the strength, and in some cases the form, of the CR. In humans, the evaluation of the UCS can be affected by a variety of different processes. These may include direct experience with the UCS or similar UCSs, social, cultural or verbally transmitted information about the UCS, and processes involved in the self-observation of responding. Factors which affect the evaluation of the UCS need not just devalue it (such as habituation might), they can also inflate its value (for instance, by individual experiences with a UCS of greater intensity than that used to train the original CR). Furthermore, processes of UCS revaluation can affect the strength of the CR independently of any changes in associative strength between CS and UCS.

This kind of model has a number of important general implications. First, it provides an associative and representational framework for human conditioning which allows it to be directly compared with findings from animal studies. Secondly, it allows a number of features unique to human conditioning to be contained in a single integrated model. These include the instructional and vicarious learning effects which may affect either the formation or strength of the CS-UCS association, or the evaluation of the UCS. Thirdly, this kind of model places some emphasis on UCS revaluation as an important process of response modulation. This is a process that has received little attention in the animal conditioning literature but is arguably of considerable importance in human conditioning (cf. Davey, 1983, 1987a). 
From the point of view of applying conditioning models to human behaviour it is important to stress that the reasons for elucidating a model of human Pavlovian conditioning are not necessarily the same as the reasons for the extensive study of Pavlovian conditioning in animals. The latter has been carried out largely as a means to understanding the nature of associative learning, and this purpose is illustrated by the sophisticated associative models that have been espoused in recent years (e.g. Rescorla and Wagner, 1972; Mackintosh, 1975; Pearce and Hall, 1980). In attempting to understand Pavlovian conditioning in humans we arguably have a much broader aim: we are driven by wider theoretical and practical considerations to attempt to understand all of those factors which affect the strength and persistence of the CR, not just the factors which affect the strength of the association between CS and UCS. The reason for this is that if conditioning theory is to be applied to the understanding of everyday human behaviour, we need a model of conditioning that makes accurate predictions about behaviour rather than some unobservable process (such as associative strength) that underlies behaviour. Clearly, the strength of a CR can be determined by many more factors than just the strength of the association between CS and UCS-UCS revaluation is just one example. One reason why traditional conditioning models of acquired fears and phobias are given such short shrift in clinical textbooks is that the principles embodied in these traditional models were designed to predict associative strenth-not behaviourand as a result they fail to explain many of the phenomena associated with phobic behaviour (e.g. Marks, 1976; Rachman. 1977). So, if contemporary models of human conditioning are to have any application to human psychological phenomena they need to be focussed more specifically on predicting behaviour. If UCS revaluation is important in modulating response strength-as it clearly appears to be-then all of the processes by which this is achieved need to be thoroughly investigated, modelled and integrated into a broader theory of human conditioning.

\section{IMPLICATIONS OF UCS REVALUATION PROCESSES FOR CONDITIONING EXPLANATIONS OF ANXIETY-BASED DISORDERS}

When it is considered that Pavlovian conditioning in humans proceeds primarily through the learning of CS-UCS associations, and that CR strength can be affected by processes of UCS revaluation, then a contemporary conditioning model of acquired fears has considerably more breadth of application than traditional contiguity-based conditioning theories.

\section{Apparent absence of trauma in clinical anamneses}

Critics of the traditional conditioning approach to phobias and fears point out that in many clinical cases there appear to have been no obvious associations between the fear-inducing situation and a traumatic event in the ontogeny of the fear. This is primarily evidenced by the failure of many phobics to recall any trauma at the time of the first appearance of their fear to the phobic stimulus (cf. Rachman, 1977; Marks, 1976). However, this is not inconsistent with the present approach. The present conditioning model conceives of acquisition of the CS-UCS association and modulation of the aversiveness of the UCS as relatively independent processes. Thus, it is quite conceivable for an individual to learn an association between a CS and UCS when the CS is relatively unaversive (a process of sensory preconditioning. cf. Prewitt, 1967; Tait, Marquis, Williams, Weinsten and Suboski, 1969; Rizley and Rescorla, 1972), and then have the aversiveness of the UCS subsequently inflated by individual experiences with a greater intensity UCS. As a result, the first CS presentation after UCS inflation will result in a relatively strong fear response to the CS (elicited by the CS's previously learnt association with the UCS). If, subsequent to UCS inflation, the CS is not followed physically by the UCS, then in such circumstances clinical anamneses are likely to provide information which misleadingly implies that the fear response to the CS was not a result of CS-UCS learning. In a laboratory analogue of this sensory preconditioning-UCS infiation scenario, White and Davey (1989) found that $S$ s learnt a differential association between a $\mathrm{CS}+$ and an innocuous $65 \mathrm{~dB}$ tone without exhibiting any differential $\mathrm{CR}$ to the $\mathrm{CS}+$. However, when the aversiveness of this tone was independently inflated by gradually increasing its intensity to $115 \mathrm{~dB}$, subsequent presentations of $\mathrm{CS}+$ alone did elicit a strong differential CR. A 'real-world' analogue of this laboratory study would be of the following kind. An individual may witness an unknown person die of a heart attack on a bus or train; on future 
occasions, riding on public transport may evoke memories of this incident but no anxiety (behaviourally-silent learning). Subsequently, however, that individual may be present when a close friend or relative dies of a heart attack, thus inflating the aversive properties of heart attacks. This may then give rise to acute anxiety when riding on public transport. However, while it can be argued that the extrapolation of laboratory studies to clinical fears needs to be independently substantiated, what these present examples clearly suggest is that a conditioning model of such fears is no longer bound by the need to discover contiguous stimulus-trauma experiences in the histories of clinical phobias.

\section{The failure to develop a phobia following traumatic experiences with a stimulus situation}

One of the perplexing difficulties with a traditional conditioning model of acquired fears is that not all individuals who have painful or traumatic experiences with a stimulus or situation actually acquire a phobic response. For instance, not all people who have painful experiences undergoing dental treatment acquire a dental phobia, not everyone who experiences a violent thunderstorm acquires a thunderstorm phobia, and not all fliers who experience a traumatic flying accident express a subsequent anxiety of flying (Lautch, 1971; Liddell and Lyons, 1978; Aitken, Lister and Main, 1981; Goorney, 1970). Clearly, if a Pavlovian account of acquired fears is to have any credence and predictive validity it must be able to specify under what conditions associative experiences with a traumatic event fail to promote a fear or phobia. There are at least two aspects of contemporary conditioning theory that attempt to account for these problematic examples; one is associative, the other is concerned with UCS revaluation.

First, if a CS is presented alone on several occasions before it is paired with an aversive UCS, it is significantly less likely to become associated with that UCS. This process is known as latent inhibition, and is found in both animal and human conditioning (cf. Siddle and Remington, 1987; Rescorla, 1969; Lubow, 1973). Applying this principle to acquired fears, an individual would be much less likely to acquire, for example, a dental phobia following a painful experience at the dentists if they had undergone painless dental treatment numerous times before. In an investigation of this possibility, Davey (1989) found that individuals who reported having a painful dental experience but did not acquire a dental anxiety reported having their first traumatic dental experiences significantly later in their dental treatment careers than individuals who did acquire a dental anxiety. Such facts are entirely consistent with the operation of latent inhibition, and provide at least one means for predicting situations in which fears may fail to develop in the wake of a traumatic associative experience.

Secondly, an individual may fail to acquire a phobia following a traumatic CS-UCS associative experience because factors other than the trauma are important contributors to evaluating the UCS. For instance, in the case of dental phobias, information provided from other sources (e.g. dental health care propaganda) may dramatically modulate the aversiveness of the pain experienced under dental treatment. Having good teeth might thus be the learned effective consequence of attending the dentists, not experiencing aversive or fear-inducing pain. However, Davey (1989) found no evidence that an individual's views on the beneficial health consequences of dental treatment actually attenuated dental anxieties, but did find some evidence that individuals who reported they were good at enduring pain were more likely to report a longer interval between their first dental treatment and their first painful dental treatment. Thus, revaluation of a painful or traumatic dental experience may not be affected by attempting to persuade the individual of the advantages of dental treatment, but may be affected by the individual's overall perception of pain in general. That is, any experience which leads to a better endurance of pain is likely to have some effect on revaluing specific painful UCSs such as painful dental treatment.

\section{Spontaneous fuctuations in the fear response}

The process of UCS revaluation permits dramatic fluctuations in fear to a CS without any intervening experience with the CS-UCS contingency, and is consistent with many of the spontaneous fluctuations in fear exhibited by phobic and neurotic individuals. One such example of the apparent spontaneous fluctuation of anxiety responses is the complete remission of symptoms known as spontaneous remission. Little is known about the exact causes of this phenomenon (cf. Rachman and Wilson, 1980), but where information is available spontaneous 
remission appears to coincide with favourable changes in the individual's environment, social status or individual relationships (e.g. Imber, Nash, Hoehn-Sark, Stone and Franks, 1968; Stevenson, 1961; Friess and Nelson, 1942). In particular circumstances, spontaneous remission can be directly attributed to the alleviation of the aversive event maintaining the neurosis (e.g. the death of burdensome or antagonistic relatives); whereas in other circumstances a significant worsening of the neurotic symptoms can be attributed to the worsening of physical conditions which reinforced neurotic tendencies (e.g. progressive organic disease accompanying increased hypochondrial and depressive trends) (cf. Friess and Nelson, 1942). Some cases of spontaneous remission also bear a striking similarity to UCS revaluation bought about by the self-observation of changes in response strength. Stevenson (1961) reports a number of instances where spontaneous remission appears to result from the individual's forced interaction with their phobic situation which in turn caused a perceived reduction in their fear. Their perceived fear reduction in turn led to increased confidence in contacts with the phobic situation because these individuals now reported believing that their phobic situation (CS) had less threatening consequences (UCS). While a variety of cases of spontaneous remission exhibit characteristics which are consistent with the operation of processes of UCS revaluation, detailed studies of spontaneous remission are long overdue and would clearly have considerable relevance to any contemporary conditioning model of acquired fears.

\section{Incubation effects}

Eysenck $(1976,1979)$ has pointed out that one of the anomalies of a conditioning theory of phobias is that following the initial conditioning episode, subsequent CS-alone presentations appear to produce successive increments in the strength of the CR even though the UCS itself is not presented. This phenomenon is quite widely reported in both the animal and clinical literature (Napalkov, 1963; Campbell, Sanderson and Laverty, 1964; Eysenck, 1968), and is clearly contrary to traditional incremental-decremental models of response strength. Nevertheless, if one considers that the strength of the CR can be affected by changes in the individual's evaluation of the UCS, then incubation phenomena are within the bounds of explanation of a conditioning account. For instance, following a conditioning episode the value of the UCS may become inflated in some way such that future CS-alone presentations evoke a stronger $C R$ as a result of this inflation. There are a number of possible ways in which this inflation could be effected. First, it is clear that following the initial conditioning episode some neurotic individuals rehearse the trauma in their minds (cf. Marks, 1987). This rehearsal, combined with the tendency of anxiety-disorder patients to focus on the aversive or traumatic contents of events (e.g. Beck, Laude and Bohert, 1974; Butler and Mathews, 1983; Mathews, 1986) could (i) lead to inflation of the aversive evaluation of the UCS prior to subsequent encounters with the CS, and (ii) single out and refine the aversive or fear-relevant aspects of the UCS for recall on future CS presentations.

A further important factor which could contribute to UCS inflation and hence to incubation-type effects is the individual's discrimination of their own fear response. Cracknell and Davey (1988) and Davey (1987a) have demonstrated that the magnitude of an aversive CR is directly related to the individual's perception of and belief in the strength of their CR and UCR. The stronger they perceive these reactions to be, or are led to believe they are as a result of false information, then the stronger and more resilient to extinction is their actual CR. Extrapolating these laboratory findings to cases of clinical incubation, individuals who exhibit incubation of a fear reaction are likely to be those who have a strong tendency to focus in on and discriminate their own physiological reactions to fearful situations, and who are likely to exaggerate their perceptions of these reactions as a result of either attentional predispositions or what they consider to be relevant information from other sources (such as their own ruminations on the possible consequences of interaction with the fear-evoking situation). Such cognitive dispositions are likely to inflate the individual's aversive evaluation of the consequences of interaction with their fear-evoking situation (nominally the UCS) and result in inflated conditioned fear reactions on subsequent encounters.

Thus, the UCS revaluation interpretation of incubation effects would suggest that incubation effects would be found most predominantly in (i) individuals who have a strong predisposition to single out the aversive or traumatic aspects of events for processing, (ii) individuals who readily 
discriminate and attend to the strength of their fear reactions, and (iii) individuals who have a tendency to overestimate the strength of their own fear reactions.

\section{IMPLICATIONS OF UCS REVALUATION PROCESSES FOR A CONDITIONING MODEL OF ACQUIRED FEARS}

In summary, contemporary models of Pavlovian conditioning in humans suggest that processes of CS-UCS association and UCS revaluation may be largely independent. An important ramification of this fact is that Pavlovian performance can be immediately and dramatically affected by factors other than those which affect the associative strength between CS and UCS. In essence, any factor which influences the individual's evaluation of the UCS will affect response strength to subsequent presentations of the CS. This has a number of implications for the assumptions supporting any conditioning model of acquired fears and enables a conditioning model to cover a variety of clinical phenomena that were hitherto problematic. For instance, (i) since the acquisition of a CS-UCS association and modulation of the aversiveness of the UCS are relatively independent processes, a conditioning model is no longer bound by the need to discover contiguous stimulus-trauma experiences in the histories of clinical phobias; (ii) there are circumstances in which a conditioning model can predict the failure to acquire a learnt fear despite traumatic experiences with a stimulus or situation; these include circumstances beneficial to the operation of latent inhibition, and circumstances in which the aversive value of the UCS is significantly attenuated following the conditioning episode; (iii) the spontaneous remission of phobic or fear symptoms is not inconsistent with a conditioning model, given that events consistent with a favourable revaluation of the consequences of interaction with the phobic event or situation (UCS) can be identified; and (iv) a model of conditioning which allows for processes which may lead to inflation of the aversive properties of the UCS following an initial conditioning episode would predict-under specified circumstances-the phenomenon of clinical incubation.

These arguments represent a preliminary attempt to construct a contemporary model of human conditioning that can be directly applied to, and make specific predictions about, the psychopathology of clinical fears. While this model already possesses characteristics which make it significantly different from traditional contiguity-based conditioning models, its main features should become clearer with further integration of our knowledge from animal conditioning and extensive study of UCS revaluation processes in humans.

Acknowledgements-The author wishes to thank Chris Brewin, Hans Eysenck, Richard Hallam, Isaac Marks, Irene Martin and Andrew Mathews for helpful comments on earlier drafts of this paper. Reprints may be obtained from Graham Davey, Psychology Division. Department of Social Science, The City University, Northampton Square, London, ECIV OHB, England.

\section{REFERENCES}

Aitken R. C. B., Lister J. A. and Main C. J. (1981) Identification of features associated with flying phobias in aircrew. Br. J. Psychiat. 139, 38-42.

Beck A. T., Laude R. and Bohert M. (1974) Ideational components of anxiety neurosis. Archs gen. Psychiat. 31, 319-325.

Butler G. and Mathews A. (1983) Cognitive processes in anxiety. Adt. Behar. Res. Ther. 5, 51-62.

Campbell D.. Sanderson R. E. and Laverty S. A. (1964) Characteristics of a conditioned response in human subjects during extinction trials following a simple traumatic conditioning trial. J. abnorm. soc. Psychol. 68, 627-693.

Cleland $G, G$. and Davey G. C. L. (1982) The effect of satiation and reinforcer devaluation on signal-centered behavior in the rat. Learn Motit. 13, 343-360.

Cracknell S. and Davey G. C. L. (1988) The effect of perceived unconditioned response strength on conditioned responding in humans. Med. Sci. Res. 16, 169-170.

Davey G. C. L. (1983) An associative view of human classical conditioning. In Animal Models of Human Behatior (Edited by Davey G. C. L.). Wiley, Chichester.

Davey G. C. L. (1987a) An integration of human and animal models of Pavlovian conditioning: associations. cognitions and attributions. In Cognitive Processes and Patlotian Conditioning in Humans (Edited by Davey G. C. L.). Wiley, Chichester.

Davey G. C. L. (1987b) Integrating human and animal theories of conditioning. J. Psychophysiol. 1, 105-108.

Davey G. C. L. (1987c) Integrating contemporary models of animal and human conditioning: implications for behaviour therapy. Paper presented to the 17th annual meeting of the European Association for Behaviour Therapy, Amsterdam.

Davey G. C. L. (1988) Pavlovian conditioning in humans: UCS revaluation and the self-observation of conditioned responding. Med. Sci. Res. 16, 957-961. 
Davey G. C. L. (1989) Dental phobias and anxieties: evidence for conditioning processes in the acquisition and modulation of a learned fear. Behav. Res. Ther. 27, 51-58.

Davey G. C. L. and Arulampalam T. (1981) Second-order electrodermal conditioning in humans. IRCS Med. Sci. 9, 567-8.

Davey G. C. L. and Arulampalam T. (1982) Second-order 'fear' conditioning in humans; persistence of CR2 following the extinction of CR1. Behar. Res. Ther. 20, 391-396.

Davey G. C. L. and McKenna I. (1983) The effect of postconditioning revaluation of CSI and UCS following Pavlovian second-order electrodermal conditioning in humans. Q. J. exp. Psychol. 35B, 125-133.

Dawson M. E. and Furedy J. J. (1976) The role of awareness in human differential autonomic classical conditioning: the necessary-gate hypothesis. Psychophysiology 13, 50-53.

Dawson M. E. and Schell A. (1987) Human autonomic classical conditioning: the role of conscious cognitive factors. In Cognitive Processes and Pavlovian Conditioning in Humans (Edited by Davey G. C. L.). Wiley, Chichester.

Dawson M. E., Catania J. J., Schell A. M. and Grings W. W. (1979) Autonomic classical conditioning as a function of awareness of stimulus contingencies. Biol. Psychol. 9, 23-40.

Dickinson A. (1980) Contemporary Animal Learning Theory. Cambridge University Press.

Eysenck H. J. (1968) A theory of incubation of anxiety/fear responses. Behar. Res. Ther. 6, 319-321.

Eysenck H. J. (1976) The learning theory model of neurosis-a new approach. Behav. Res. Ther. 14, 215-267.

Eysenck H. J. (1979) The conditioning model of neurosis. Behav. Brain Sci. 3, 155-199.

Friess C. and Nelson M. J. (1942) Psychoneurotics five years later. Am. J. Med. Sci. 203, 539-558.

Goorney A. B. (1970) Treatment of aviation phobias by behaviour therapy. Br. J. Psychiat. 117, 535-544.

Grings W. W. (1973) Cognitive factors in electrodermal conditioning. Psychol. Bull. 79, 200-210.

Holland P. C. and Rescorla R. A. (1975) The effects of two ways of devaluing the unconditioned stimulus after first- and second-order appetitive conditioning. J. exp. Psychol. Anim. Behat. Proc. 1, 355-363.

Holland P. C. and Straub J. J. (1979) Differential effects of two ways of devaluing the unconditioned stimulus after Pavlovian appetitive conditioning. J. exp. Psychol. Anim. Behav. Proc. 5, 65-78.

Imber S., Nash E., Hoehn-Sark R., Stone A. and Franks J. L. (1968) A 10-year follow-up of treated psychiatric outpatients. In An Evaluation of the Results of Psychotherapies (Edited by Lesse S.). Springtield, IU.

Lautch H. (1971) Dental phobia. Br. J. Psychiat. 119, 151-158.

Liddell A. and Lyons M. (1978) Thunderstorm phobias. Behav. Res. Ther. 16, 306-308.

Lubow R. E. (1973) Latent inhibition. Psychol. Bull. 79, 398-407.

Mackintosh N. J. (1975) A theory of attention; variations in the associability of stimuli with reinforcement. Psychol. Rev. 82, 276-298.

Marks I. M. (1976) Clinical phenomena in search of laboratory models. In Psychopathology: Experimental Models (Edited by Maser J. D. and Seligman M. E. P.). Freeman, San Francisco.

Marks I. M. (1987) Fears, Phobia and Rituals. Oxford University Press, New York.

Mathews A. (1986) Cognitive processes in anxiety and depression: discussion paper. J. R. Soc. Med. 79, 158-161.

Napalkov S. V. (1963) Information processing and the brain. In Progress in Brain Research (Edited by Weiner N. and Schodel J.), Vol. 2., pp. 59-69.

Pearce J. M. and Hall G. (1980) A model for Pavlovian learning: variations in the effectiveness of conditioned but not of unconditioned stimuli. Psychol. Rev. 87, 532-552.

Prewitt E. P. (1967) Number of preconditioning trials in sensory preconditioning using CER training. J. comp. Physiol. Psychol. 64, 360-362.

Rachman S. (1977) The conditioning theory of fear-acquisition; a critical examination. Behat. Res. Ther. 15, $375-387$.

Rachman S. and Wilson G. P. (1980) The Effects of Psychotherapy. Freeman, San Francisco.

Rescorla R. A. (1969) Pavlovian conditioned inhibition. Psychol. Bull. 72, 77-94.

Rescorla R. A. (1973) Second-order conditioning; implications for theories of learning. In Contemporary Approaches to Learning and Conditioning (Edited by McGuigan F. J. and Lumsden D.). Winston, New York.

Rescorla R. A. (1977) Pavlovian second-order conditioning: some implications for instrumental behavior. In Operant Patlotian Interactions (Edited by Davis H. and Hurwite H. M. B.). Erlbaum, New Jersey.

Rescorla R. A. (1980) Patlocian Second-order Conditioning: Studies in Associatite Learning. Erlbaum, New Jersey.

Rescorla R. A. and Wagner A. (1972) A theory of Pavlovian conditioning: variations in the effectiveness of reinforcement and nonreinforcement. In Classical Conditioning II: Current Research and Theory (Edited by Black A. H. and Prokasy W. F.). Appleton, New York.

Rizley R. C. and Rescorla R. A. (1972) Associations in second-order conditioning and sensory preconditioning. J. comp. Physiol. Psychol. 81, $1-11$.

Siddle D. A. T. and Remington B. (1987) Latent inhibition and human Pavlovian conditioning: research and relevance. In Cognitive Processes and Pavlovian Conditioning in Humans (Edited by Davey G. C. L.). Wiley, Chichester.

Stevenson I. (1961) Process of spontaneous recovery from the psycho-neuroses. Am. J. Psychiat. 117, 1057-1064

Tait R. W., Marquis H. A., Williams R., Weinsten L. and Suboski M. D. (1969) Extinction of sensory preconditioning using CER training. J. comp. Physiol. Psychol. 69, 170-172.

White K. and Davey G. C. L. (1989) Sensory preconditioning and UCS inflation in human 'fear' conditioning. Behav. Res. Ther. 27, 161-166. 